\title{
Low-Molecular-Weight Heparin versus Unfractionated Heparin in Pregnant Women with Recurrent Abortion associated with Antiphospholipid Syndrome
}

\author{
Abdel-Qader Farag Abdel-Qader, Farid Ibrahim Hassan, Mofeed Fawzy Mohammad \\ Department of Obstetrics and Gynaecology, Faculty of Medicine, Alazhar University, Cairo, Egypt
}

Corresponding auther: Abdel - Qader Farag Abdel - Qader

Email: dr.abdelqader.egypt@gmail.com

\begin{abstract}
Objectives: to compare the efficacy and safety of the combination of unfractionated heparin (UFH) and Low Dose Aspirin (LDA) with that of low-molecular-weight heparin (LMWH) and LDA in the management of pregnant women with recurrent pregnancy loss secondary to antiphospholipid syndrome (APS). Methods : In a randomized prospective study, 60 women with a history of 3 or more consecutive spontaneous abortions and positive antiphospholipid antibodies were assigned in equal numbers to receive either UFH (5000 units, twice daily) plus LDA, or LMWH (enoxaparin 40 $\mathrm{mg}$, once daily) plus LDA as soon as pregnancy was diagnosed. Results: Twenty-four women in the LMWH group (80\%) compared to 20 women in the UFH group $(66.67 \%$ ) delivered a viable infant (p $>0.05$ ). There was no significant difference in pregnancy complications or neonatal morbidity between the 2 groups. There were no incidences of excessive bleeding, thrombocytopenia, or osteoporotic fractures of excessive bleeding, thrombocytopenia, or osteoporotic fractures in either group. Conclusion: In this study, the use of LDA in combination with LMWH during pregnancy for the prevention of recurrent pregnancy loss in women with antiphospholipid syndrome seems to be as safe as UFH plus LDA. Large randomized trials will be required to determine differences in outcome with LMWH and LDA compared with treatment with UFH combined with LDA in this group of patients.
\end{abstract}

Keywords: recurrent pregnant loss, antiphospholipid antibodies, low molecular weight heparin, unfractionated heparin

\section{Introduction}

The antiphospholipid syndrome (APS) is an autoimmune disease characterized by recurrent arterial or venous thrombosis, pregnancy morbidity and the persistence of positive antiphospholipid antibodies (1). Antiphospholipid syndrome may occur either isolated (primary syndrome) ${ }^{(2)}$, or in the setting of an underlying disease, mainly systemic lupus erythematosus (secondary syndrome) ${ }^{(3)}$.

Women with antiphospholipid syndrome (APS) have live birth rates as low as $10 \%$ to $50 \%$ in pregnancy without pharmacological treatment. Fetal losses in APS have been attributed to thrombosis of the uteroplacental vasculature, placental infarction. Not surprisingly, therapy for pregnant women with APS is now focused on preventing thrombosis at the maternal-fetal interface ${ }^{(4)}$.

Although low molecular weight heparin is more expensive than unfractionated heparin, low molecular weight heparin has the advantage over unfractionated heparin of a longer plasma half-life and a more predictable dose response and therefore the potential for once daily administration. Furthermore, low molecular weight heparin causes less heparin induced thrombocytopenia and heparin-induced osteoporosis $^{(5)}$.

\section{Patients and Methods}

The present study was a 2-arm, prospective, randomized controlled trial of 60 women with a history of 3 or more consecutive pregnancy losses before 10 weeks of gestation and positive antiphospholipid antibodies on 2 or more occasions at least 12 weeks apart. The study was performed at Al-Azhar University Hospitals (Al-Hussin and Sayed Galal Hospitals), Cairo, Egypt. In the period from 1 Jan to 1 Sep 2018 .The study protocol was approved by the ethics committee of the hospital, the women were counseled about the benefits and risks of heparin and aspirin therapy, and informed consent was obtained from all participants.

We evaluated data from 242 women who were complaining of recurrent abortion to identify those who could be included in the study. 
The inclusion criteria:

1. History of 3 or more consecutive spontaneous abortions before 10 weeks of gestation, and positive lupus anticoagulant (LAC) and/or anticardiolipin antibodies (IgG and $\operatorname{IgM}$ ) on 2 or more occasions at least 12 weeks apart.

2. Age between 18 and 37 years.

3. Body mass index (BMI, calculated as weight in kilograms divided by the square of height in meters) between 19 and 29 .

\section{The exclusion criteria:}

1. From history:

a) Pervious venous or arterial thrombotic episodes.

b) Diabetes mellitus.

c) Kidney or liver disease.

d) Gastric ulcer.

e) Sensitivity to aspirin, UFH, or LMWH.

2. By investigations:

a) Paternal chromosomal abnormalities.

b) Uterine malformation detected by hysterosalpigography.

c) Cervical incompetence.

d) Luteal phase defect.

e) Hyperprolactinemia.

f) Polycystic ovary syndrome.

g) Abnormal thyroid functions.

Serum IgG and IgM anticardiolipin antibodies were assayed by using a commercially available standardized enzyme linked immnunosorbent assay and positive $\operatorname{IgG}$ and $\operatorname{IgM}$ values were defined as more than 40 GPL units and more than 40 MPL units. respectively, An activated partial thromboplastin time test and a dilute Russell viper venom test were performed to screen for LAC. If either of these tests was prolonged, the presence of LAC was documented by mixing and confirmatory tests.

The 60 women who fulfilled the inclusion criteria and enrolled in the study were randomly allocated to 1 of 2 groups via a computer generated randomization list and sequentially numbered, opaque sealed envelopes, each containing the allocation information written on a card. The computer generated randomization list and the sealed envelopes were prepared by a statistician not involved in the study. The envelopes were opened sequentially by a staff nurse to assign the women to receive either UFH plus LDA $(n=30)$ or LMWH plus LDA $(n=30)$.
Administration of prenatal vitamins and combined oral calcium and vitamin D3 (600 mg and $400 \mathrm{IU}$, respectively, once) was started before conception and continued throughout pregnancy. Administration of LDA (75mg, once daily [aspocid paediatric; chemical industries development, Giza, Egypt]) was started before conception and continued through pregnancy until 36 weeks of gestation. Anticoagulation treatment was started as soon as the serum pregnancy test became positive. The women in the UFH group received heparin calcium (5000 $\mathrm{U}$ subcutaneously, twice daily [cal-heparin; Amoun pharmaceutical, Cairo, Egypt]) and the women in the LMWH group received enoxaparin (40 mg subcutaneously, once daily [Clexan; Sanofi-Aventis. Paris, France]).

Fetal viability was confirmed by transvaginal ultrasound at 6 weeks of gestation. Prenatal checkup was carried out every 2 weeks from enrollment to 32 weeks of gestation, then weekly until delivery. Serial ultrasound examination at 2 week intervals was started at 24 weeks of gestation to monitor fetal growth. To detect the development of thrombocytopenia, the platelet count was measured before starting therapy was started, then every 2 weeks for the first 2 months, followed by every 4 weeks. Heparin therapy was discontinued if the platelet count was less than $100000 / \mathrm{mL}$ or if there was $\geq 50 \%$ drop in the baseline platelet count.

\section{Statistical Analysis}

Statistical Analysis was performed via t, mann-whitney $\mathrm{u}$, and $\mathrm{x} 2$ test, as appropriate . a yates correction equation was used when the expected frequency was less than 5. $\mathrm{P}<0.05$ was considered to be statistically significant . all statistical calculations were performed using excel version 7 (Microsoft, new york, NY USA) and SPSS (SPSS, Chicago, IL, USA)

Data were tabulated, coded then analyzed using the computer program: "SPSS (Statistical package for social science) version 17.0"

\section{To obtain:}

Descriptive data:

Descriptive statistics were calculated in the form of:

1. Mean \pm Standard deviation (SD) for quantitative parametric data. 
2. Frequency (Number-percent) for qualitative data.

\section{Analytical statistics:}

In the statistical comparison between the different groups, the significance of difference was tested using one of the following tests:

1- Student's t-test:-Used to compare between mean of two groups of numerical (parametric) data.
2- Inter-group comparison of categorical data was performed by using either pearson'schi square test (X2-value) or fisher exact test.

* Odds ratio (OR) with Confidence interval 95 $\%$ ( CI 95\%) was calculated to detect risk.

* A P value $<0.05$ was considered statistically significant in all analyses.

\section{Results}

Table (1): Baseline characteristics of study participants

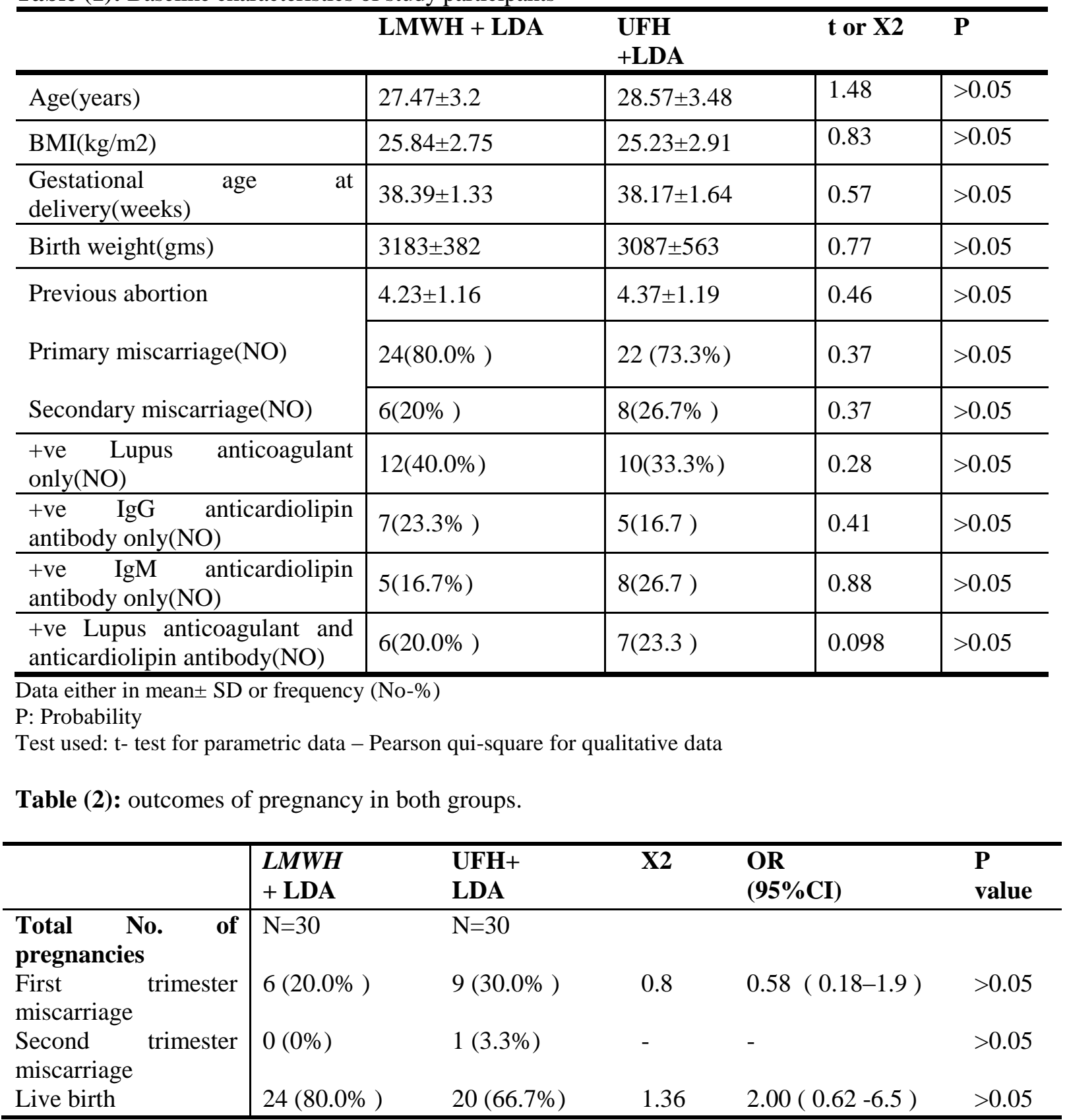


Table (3): Impact of treatment on gestational age at delivery and birth weight in both groups.

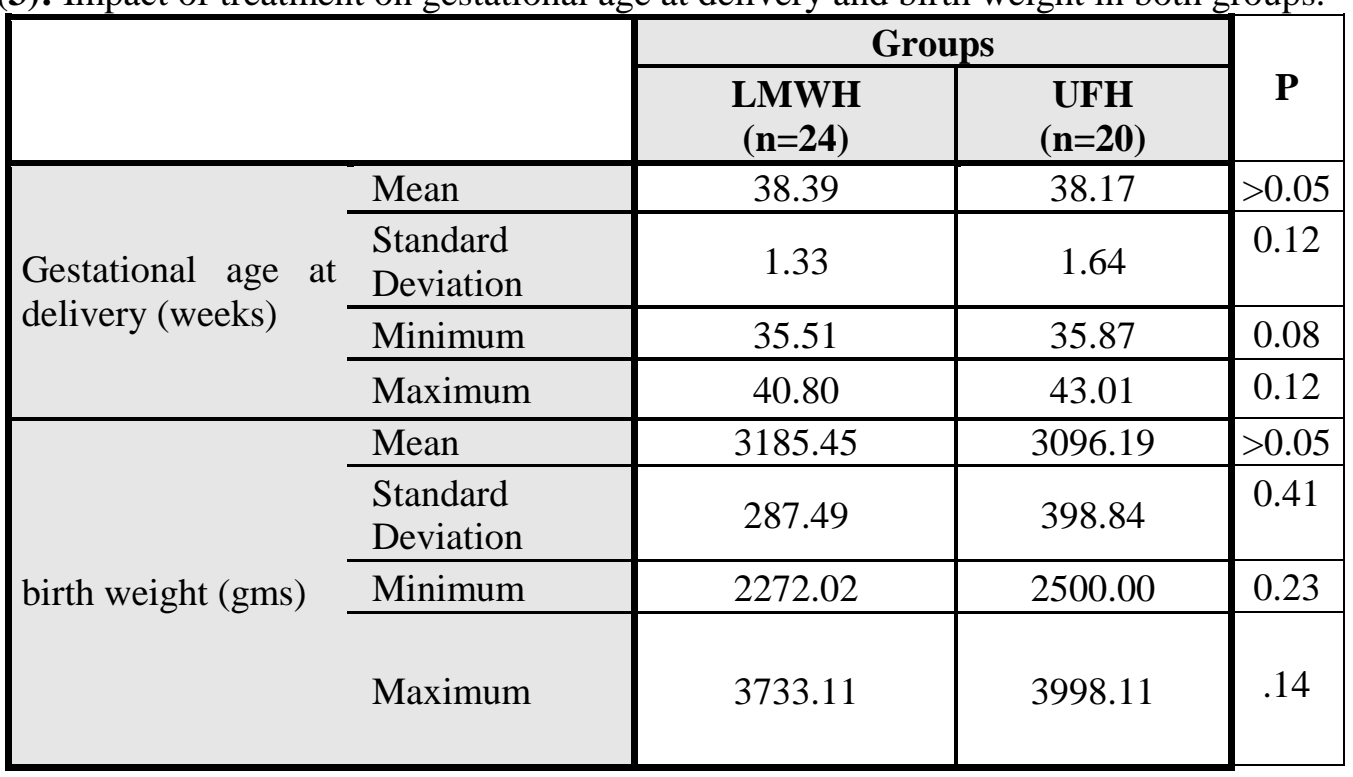

Table (4): Non Obstetrics complications in both groups.

\begin{tabular}{l|lllll}
\hline & $\begin{array}{l}\text { LMWH } \\
+L D A\end{array}$ & $\begin{array}{l}\text { UFH+ } \\
\text { LDA }\end{array}$ & $X 2$ & $\begin{array}{l}\text { OR } \\
(95 \% C I)\end{array}$ & P value \\
\hline & & & & & - \\
Osteoporotic fractures & $0(0 \%)$ & $0(0 \%)$ & - & - & - \\
Thrombocytopenia & $0(0 \%)$ & $0(0 \%)$ & - & - & $1.00(0.18-5.4)$ \\
Subcutaneous bruises & $3(10.0 \%)$ & $3(10.0 \%)$ & 0 & $1.00(0.18)$ & 1.00 \\
Skin allergy & $0(0 \%)$ & $1(3.3 \%)$ & - & - & \\
\hline
\end{tabular}

Table (5): Late obstetric complications during pregnancy in both groups

\begin{tabular}{l|lllll}
\hline & $L M W H+L D A$ & $\begin{array}{l}\text { UFH+ } \\
\text { LDA }\end{array}$ & X2 & $\begin{array}{l}\text { OR } \\
(95 \% C I)\end{array}$ & $\begin{array}{l}P \\
\text { value }\end{array}$ \\
\hline & $\mathrm{N}=24$ & $\mathrm{~N}=20$ & & & \\
& $2(8.3 \%)$ & $1(5.0 \%)$ & - & $1.7(0.15-20.6)$ & 1.00 \\
Pre-eclampsia & $1(4.2 \%)$ & $2(10.0 \%)$ & - & $0.39(0.03-4.66)$ & $>0.05$ \\
IUGR & $3(12.5 \%)$ & $2(10.0 \%)$ & - & $1.29(0.19-8.6)$ & 1.00 \\
Preterm labor & $0(0 \%)$ & $0(0 \%)$ & - & - & \\
IUFD & & & & & - \\
Obst. Hge. & $0(0 \%)$ & $0(0 \%)$ & - & \\
\hline
\end{tabular}

\section{Discussion}

In the present study, LMWH plus LDA treatment resulted in a slightly higher live birth rate $(80 \%)$ in women with recurrent spontaneous abortion secondary to APS

Despite the high cost of LMWHs, they have several advantages over UFH. In contrast to UFH, LMWHs have lower anti-IIa activity than anti-Xa activity, resulting in a lower risk of bleeding at similar levels of anticoagulation.
LMWHs also have a longer half-life, and more stable and predictable pharmacokinetics, and therefore can be administrated once daily without the need for monitoring ${ }^{(6)}$. There is evidence that compared with UFH, LMWHs are associated with a lower risk of excessive bleeding, heparin induced osteoporosis, and thrombocytopenia ${ }^{(3)}$.Furthermore, in contrast to warfarin no teratogenic effects or neonatal bleeding episodes have been reported because 
LMWHs do not cross the placental barrier ${ }^{(8)}$.These advantages make LMWHs the anticoagulant of choice during pregnancy ${ }^{(3)}$.

In the present study, the live birth rate was $80 \%$ in the LMWH plus LDA group and $66.7 \%$ in the UFH plus LDA group. This deference was not significant because of the small sample size of both groups. To detect a significant difference in favor of LMWH plus LDA, a sample size of 410 women would have had to be recruited to achieve a study power of $80 \%$ at a significance level of 0.05 (2-tailed). The main limitation of the present study was the small sample size. The aim of the present study was to compare the efficacy and safety of LMWH plus LDA with those of UFH plus LDA, and the results of the study could be used in future meta-analyses comparing both management options. A further was that the trial was not blind because it was considered unethical to give women in the LMWH group a once daily placebo injection until the end of pregnancy ${ }^{(5)}$.

In conclusion, the results of the present study indicate that the efficacy and safety of LMWHs plus LDA in the management of women with recurrent spontaneous abortion secondary to APS were superior or at least equivalent to those of UFH plus LDA. The results highlight the need for a larger randomized controlled trial to determine whether LMWH plus LDA should be the treatment of choice for women with recurrent abortion secondary to APS.

\section{Conclusion}

LMWH plus LDA was successfully used as an alternative to UFH plus LDA in the management of recurrent abortion secondary to APS. The results highlight the need for a larger randomized controlled trial to determine whether LMWH plus LDA should be the treatment of choice for recurrent abortion secondary to APS.

\section{References}

1.Hughes GR( 2014): Thrombosis, abortion, cerebral disease and the lupus anticoagulant. BMJ.,287:1088-1089

2.Asherson RA, 2010. A primary' antiphospholipid syndrome? Journal of Rheumatology; 15: 1742-1746

3.Alarcon-Segovia D, Deleze M, Oria CV, Sanchez-Guerrero J, G6mez- Pacheco L, Cabiedes $\mathbf{J}$ et al.(2012): Antiphospholipid antibodies and the antiphospholipid syndrome in systemic lupus erythematosus. Medicine, 68: 353365.

4.Asherson RA (2010): A primary' antiphospholipid syndrome? Journal of Rheumatology, 15: 1742-1746.

5.Casele HL(2013): The use of unfractionated heparin and low molecular weight heparins in pregnancy. Clin Obstet Gynecol.,49 (4):895-905.

6.Derksen RH, Khamashta MA, Branch DW (2014): Management of the obstetric antiphospholipid syndrome.Arthritis Rheum., 50: 1028-1039.

7.Fouda UM, Sayed AM, Abdon AM, Ramadan DI, Fonda IM, Zaki MM(2011):Enoxaparin versus unfractionated heparin in the management of recurrent abortion secondary to antiphospholipid syndrome. International Journal of Gynecology and obstetrics., 112 :211-215.

8.Harenberg J, Schneider D, Hellman L, Wolf H (2014): Lack of anti-factor Xa activity in umbilical cord vein samples after subcuataneous administration of heparin or low molecular weight heparin in pregnant women. Thromb Haemost., 23:314-320. 